\title{
Hall effects on hydromagnetic Couette flow of Class-II in a rotating system in the presence of an inclined magnetic field with asymmetric heating/cooling of the walls
}

\author{
G. S. Seth ${ }^{1 *}$ and S. M. Hussain ${ }^{2}$ \\ ${ }^{1 *}$ Department of Applied Mathematics, Indian School of Mines Dhanbad, INDIA \\ ${ }^{2}$ Department of Mathematics, O. P, Jindal Institute of Technology Raigarh, INDIA \\ "Corresponding Author: e-mail: gsseth_ism@yahoo.com Tel.+91-326-2235421.Fax.+91-326-2296619
}

\begin{abstract}
Hall effects on steady hydromagnetic Couette flow of class-II of a viscous, incompressible and electrically conducting fluid with non-conducting walls in a rotating system in the presence of an inclined magnetic field is investigated. Exact solution of the governing equations is obtained in closed form. Expressions for the shear stress at the moving plate due to primary and secondary flows and mass flow rates in the primary and secondary flow directions are also derived. Asymptotic behavior of the solution for velocity and induced magnetic field is analyzed, for small and large values of rotation parameter $K^{2}$ and magnetic parameter $M^{2}$ to gain some physical insight into the flow pattern. Heat transfer characteristics of the fluid are considered taking viscous and Joule dissipations into account when walls of the channel are asymmetrically heated or cooled. Numerical solution of energy equation and numerical values of rate of heat transfer at the stationary and moving plates are computed with the help of MATLAB software. The numerical values of velocity, induced magnetic field and fluid temperature are displayed graphically versus channel width variable $\eta$ for various values of Hall current parameter $m$ and angle of inclination of magnetic field $\theta$ whereas numerical values of shear stress at the moving plate due to primary and secondary flows, mass flow rates in the primary and secondary flow directions and rate of heat transfer at the stationary and moving plates are presented in tabular form for various values of $m$ and $\theta$.
\end{abstract}

Keywords: Inclined Magnetic field, Coriolis force, Hall current, Viscous and Joule Dissipations.

DOI: http://dx.doi.org/10.4314/ijest.v3i8.19

\section{Introduction}

Theoretical /experimental investigation of fluid flow in a rotating environment is of much significance due to occurrence of various natural phenomena and its application in various technological situations which are directly governed by the action of Coriolis force. The broad subject of oceanography, meteorology, atmospheric science and limnology contain some important and essential features of rotating fluids. Large scale circulation in the atmosphere and oceans, construction of turbines and other centrifugal machines are some of the areas of application of rotating fluids. Rotating fluids have an intrinsic stability in the sense that if a fluid particle is displaced from its equilibrium position of the rigid body rotation, the Coriolis force acts as a restoring force. The current interest in the study of magnetohydrodynamics of rotating fluids is motivated by several important problems, namely, maintenance and secular variations of the earth's magnetic field, the internal rotation rate of the sun, the structure of rotating magnetic stars, the planetary and solar dynamo problems and centrifugal machines (rotating hydromagnetic generator, rotating-drum separators for liquid-metal MHD applications etc).

The problem of MHD Couette flow of an electrically conducting fluid in a rotating system in the presence of a magnetic field is investigated by many scientists and engineers due to its varied and wide applications in the areas of geophysics, astrophysics and 
fluid engineering viz. in the design of nuclear power reactors, heat exchangers utilizing liquid metal coolant, MHD power generators, MHD pumps, MHD accelerators, underground water energy storage system etc. It is well known that the theory of Couette flow is used for the measurement of viscosity and estimating drag force in many wall driven devices. There exist two types of problems in science and engineering when a vast expanse of fluid is bounded by a solid body e.g. plate (Batchelor, 1988) which are mentioned below.

Fluid flow of Type-I: Fluid flow induced by movement of a plate when the fluid at infinity i.e. fluid outside the boundary layer region, is stationary. Schematic diagram of fluid flow of Type-I is mentioned in Figure 1a.

Fluid flow of Type-II: Fluid flows past a stationary plate which is induced due to movement of fluid at infinity i.e. fluid outside the boundary layer region. The fluid outside the boundary layer region is called free stream and velocity related to it is called free stream velocity. Schematic diagram of fluid flow of Type-II is mentioned in Figure $1 \mathrm{~b}$.

Taking into account the nature of the above mentioned fluid flows we are of opinion that there are two types of MHD Couette flow (Seth and Singh, 2011, Seth et al., 2011) viz. (i) MHD Couette flow of class-I and (ii) MHD Couette flow of class-II. The fluid flow induced due to movement of a plate when the fluid is bounded by a stationary plate, placed at a finite distance from the moving plate, is recognized as MHD Couette flow of class-I. This fluid flow is similar to above mentioned fluid flow of Type-I. The fluid flow past a stationary plate which is induced due to movement of a plate, placed at a finite distance from the stationary plate, is named as MHD Couette flow of class-II. This fluid flow is similar to above mentioned fluid flow of Type-II. It is noticed that MHD Couette flow of class-I and MHD Couette flow of class-II reduce to fluid flow of Type-I and Type-II respectively when the distance $L$ between two plates becomes very large i.e. $L \rightarrow \infty$.

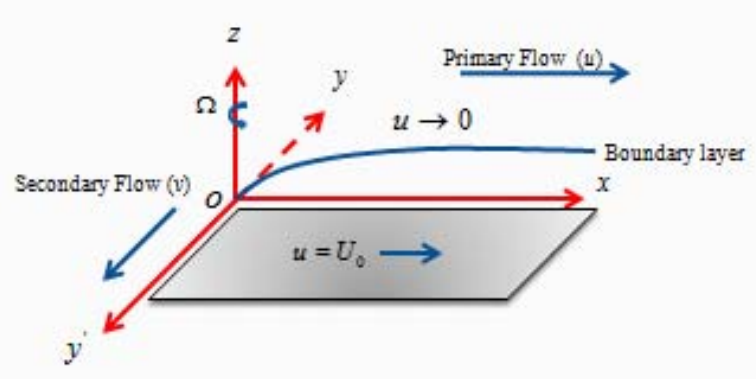

Figure 1a Schematic diagram of fluid flow of Type-I



Figure 1b Schematic diagram of fluid flow of Type-II

Mazumder (1991) initiated the study of unsteady hydrodynamic Couette flow of class-II in a rotating system. Subsequently this problem is investigated by Ganapathy (1994) and Das et al (2008) by considering different aspects of the problem. Chandran et al. (1993), Singh et al. (1994), Guchhait et al. (2011) and Jha and Apere (2011) studied MHD Couette flow of class-I in a rotating system whereas Singh(2000), Hayat et al. (2004a, 2004b), Seth and Singh (2012) and Prasad and Kumar (2012) investigated MHD Couette flow of class-II in a rotating system in the presence of a transverse magnetic field by considering different aspects of the problem. In all these investigations on MHD Couette flow, induced magnetic field produced by fluid motion is negligible in comparison to the applied magnetic field. This assumption is valid because magnetic Reynolds number is very small for metallic liquids and partially ionized fluids (Cramer and Pai, 1973). Although for the problems of geophysical and astrophysical interest and in so many MHD devices viz. MHD power generators, MHD pumps, plasma flow in accelerators etc magnetic Reynolds number is not very small so induced magnetic field cannot be neglected. It plays an important role in determining the flow features of such fluid flow problems. Keeping in view of this fact Jana et al. (1977) studied MHD Couette flow of class-I of a viscous, incompressible and electrically conducting fluid in a rotating system in the presence of a uniform transverse magnetic field taking induced magnetic field into account when the stationary plate of the channel is perfectly conducting and the moving plate of the channel is electrically non-conducting. Seth and Maiti (1982) considered this problem when both plates of the channel are electrically non-conducting.

Seth and Singh (2011) investigated MHD Couette flow of class-II of a viscous, incompressible and electrically conducting fluid in a rotating system in the presence of a uniform transverse magnetic field taking induced magnetic field into account when plates of the channel are electrically non-conducting and are heated / cooled asymmetrically whereas Seth et al (2011) studied this problem when the stationary plate of the channel is perfectly conducting and the moving plate of the channel is electrically nonconducting. In all these investigations, magnetic field is applied parallel to the axis of rotation. However, magnetic field may be inclined in certain problems of geophysical and astrophysical interest and fluid engineering e.g. in MHD power generator and magnetic material processing flow control magnetic field may act obliquely to the flow. Keeping in view of this fact, Guria et al. (2009), Bég et al. (2010), Seth et al. (2012) and Chauhan and Agrawal (2012), studied MHD Couette flow of class-I of a viscous, incompressible and electrically conducting fluid in a rotating system in the presence of an inclined magnetic field. It is worthy to note that, in an ionized fluid, where density is low and/or magnetic field is strong the effects of Hall current become significant as mentioned by Sutton and Sherman (1965). Both Hall current and rotation induce secondary flow in the fluid. Therefore, it seems to be important to compare and contrast the effects of these two agencies and also to study their combined effects. Hall current and 
rotation are likely to be important in many engineering applications viz. MHD power generators, MHD pumps and plasma flow in accelerators, geophysical and astrophysical problems of interest as well as in flows of plasmas in laboratory. Sherman and Sutton (1962) considered the effects of Hall current on the efficiency of MHD power generator. Taking into consideration the importance of such study Chauhan and Agrawal (2012), studied the effects of Hall current on steady MHD Couette flow of class-I in a rotating system in the presence of a uniform inclined magnetic field taking induced magnetic field into account.

Aim of the present investigation is to study the effects of Hall current on the problem studied by Seth and Singh (2011) in the presence of an inclined magnetic field. The significance of such fluid problems lies in the behavior of an inclined magnetic field subject to interplay of magnetic and Coriolis forces taking into account the effects of Hall current. Such an investigation has to the authors' knowledge thus far not received attention, despite its important applications in some MHD devices e.g. in rotating hybrid MHD energy generators (Bég et al., 2010).

\section{Formulation of the Problem and its Solution}

Consider steady Couette flow of a viscous, incompressible and electrically conducting fluid between electrically non-conducting parallel plates $z=0$ and $z=L$ in the presence of an inclined magnetic field $B_{0}$ which makes an angle $\theta$ with the positive direction of z-axis in xz- plane. Both the fluid and channel rotate in unison with uniform angular velocity $\Omega$ about z-axis. Flow within the channel is induced due to movement of upper plate $z=L$ with uniform velocity $U_{0}$ in $x$-direction whereas lower plate $z=0$ is kept fixed. The plates of the channel are heated /cooled asymmetrically. The schematic diagram of the physical problem is presented in Figure 1c. Since plates of the channel are of infinite extent in $x$ and $y$ directions and fluid flow is steady so all physical quantities, except pressure, depend on $z$ only. Therefore, the fluid velocity $\vec{q}$ and magnetic induction vector $\vec{B}$ are assumed in the following form

$$
\vec{q}=\left(u_{x}, u_{y}, 0\right) \text { and } \vec{B}=\left(B_{x}{ }^{\prime}+B_{0} \sin \theta, B_{y}{ }^{\prime}, B_{0} \cos \theta\right) \text {, }
$$

The assumptions of $\vec{q}$ and $\vec{B}$ are compatible with the fundamental equations of magnetohydrodynamics in a rotating frame of reference.



Figure 1c Schematic diagram of the physical problem

Taking into account the assumptions made above the governing equations for steady fluid flow of a viscous, incompressible and electrically conducting fluid in a rotating frame of reference are

$$
\begin{aligned}
& -2 \Omega u_{y}=-\frac{1}{\rho} \frac{\partial p}{\partial x}+v \frac{d^{2} u_{x}}{d z^{2}}+\frac{B_{0} \cos \theta}{\rho \mu_{e}} \frac{d B_{x}{ }^{\prime}}{d z}, \\
& 2 \Omega u_{x}=-\frac{1}{\rho} \frac{\partial p}{\partial y}+v \frac{d^{2} u_{y}}{d z^{2}}+\frac{B_{0} \cos \theta}{\rho \mu_{e}} \frac{d B_{y}{ }^{\prime}}{d z}, \\
& 0=-\frac{1}{\rho} \frac{\partial p}{\partial z}, \\
& 0=\mu_{e} \sigma B_{0} \cos \theta \frac{\partial u_{x}}{\partial z}+\frac{\partial^{2} B_{x}{ }^{\prime}}{\partial z^{2}}+m \cos \theta \frac{\partial^{2} B_{y}{ }^{\prime}}{\partial z^{2}}, \\
& 0=\mu_{e} \sigma B_{0} \cos \theta \frac{\partial u_{y}}{\partial z}+\frac{\partial^{2} B_{y}{ }^{\prime}}{\partial z^{2}}-m \cos \theta \frac{\partial^{2} B_{x}{ }^{\prime}}{\partial z^{2}},
\end{aligned}
$$


where $m=\omega_{e} \tau_{e}, \rho, v, \mu_{e}, \sigma$ and $p$ are, respectively, Hall current parameter, fluid density, kinematic coefficient of viscosity, magnetic permeability, electrical conductivity of the fluid and modified pressure including centrifugal force. $\omega_{e}$ and $\tau_{e}$ are cyclotron frequency and electron collision time respectively.

The boundary conditions for fluid velocity are

$$
\begin{aligned}
& u_{x}=0, u_{y}=0 \text { at } z=0, \\
& u_{x}=U_{0}, u_{y}=0 \text { at } z=L .
\end{aligned}
$$

Since walls of the channel are considered electrically non-conducting so the boundary conditions for induced magnetic field are

$$
\begin{aligned}
& B_{x}{ }^{\prime}=B_{y}{ }^{\prime}=0 \text { at } z=0, \\
& B_{x}{ }^{\prime}=B_{y}{ }^{\prime}=0 \text { at } z=L .
\end{aligned}
$$

Equation (4) shows that modified pressure $p$ is independent of $z$, so the values of pressure gradient terms $-\frac{1}{\rho} \frac{\partial p}{\partial x}$ and $-\frac{1}{\rho} \frac{\partial p}{\partial y}$, which are present in the equations (2) and (3), are either evaluated by using boundary conditions for the fluid velocity and induced magnetic field at the stationary plate i.e. boundary conditions (7a) and (8a) or the boundary conditions for the fluid velocity and induced magnetic field at the moving plate i.e. boundary conditions (7b) and (8b).

Using boundary conditions (7a) and (8a) in equations (2) and (3), we obtain

$$
-\frac{1}{\rho} \frac{\partial p}{\partial x}=0,-\frac{1}{\rho} \frac{\partial p}{\partial y}=0 .
$$

Using boundary conditions (7b) and (8b) in equations (2) and (3), we obtain

$$
-\frac{1}{\rho} \frac{\partial p}{\partial x}=0,-\frac{1}{\rho} \frac{\partial p}{\partial y}=2 \Omega U_{0} .
$$

Seth and Maiti (1982) investigated steady MHD Couette flow in a rotating system with electrically conducting walls in the presence of a uniform transverse magnetic field taking into account the values of pressure gradient terms mentioned in equation (9a). Such type of fluid flow problem may be regarded as MHD Couette flow of class-I. Seth and Singh (2011) considered steady MHD Couette flow in a rotating system with electrically non-conducting walls in the presence of a uniform transverse magnetic field taking into consideration the values of pressure gradient terms mentioned in equation (9b). Such type of fluid flow problem may be named as MHD Couette flow of class-II. Chauhan and Agrawal (2012) studied effects of Hall current on steady MHD Couette flow of class-I in a rotating system in the presence of an inclined magnetic field. For MHD Couette flow of class-I, equations (2) to (6) with the use of equation (9a) is presented by Chauhan and Agrawal (2012) for clear fluid region. In the absence of Hall current (i.e. $m=0$ ) and angle of inclination of magnetic field (i.e. $\theta=0$ ) equations (2) to (6) reduce to those equations which are obtained by Seth and Singh (2011).

Our problem belongs to MHD Couette of class II. Therefore, equations (2) and (3) with the use of equation (9b) reduce to

$$
\begin{aligned}
& -2 \Omega u_{y}=v \frac{d^{2} u_{x}}{d z^{2}}+\frac{B_{0} \cos \theta}{\rho \mu_{e}} \frac{d B_{x}{ }^{\prime}}{d z}, \\
& 2 \Omega\left(u_{x}-U_{0}\right)=v \frac{d^{2} u_{y}}{d z^{2}}+\frac{B_{0} \cos \theta}{\rho \mu_{e}} \frac{d B_{y}{ }^{\prime}}{d z} .
\end{aligned}
$$

Introducing dimensionless variables

$$
\eta=\frac{z}{L}, u=\frac{u_{x}}{U_{0}}, v=\frac{u_{y}}{U_{0}}, B_{x}=\frac{B_{x}{ }^{\prime}}{B_{0}}, B_{y}=\frac{B_{y}{ }^{\prime}}{B_{0}},
$$

the equations (5), (6), (10) and (11), in dimensionless form, become

$$
\begin{aligned}
& 0=\frac{d u}{d \eta}+\frac{1}{R_{m} \cos \theta} \frac{d^{2} B_{x}}{d \eta^{2}}+\frac{m}{R_{m}} \frac{d^{2} B_{y}}{d \eta^{2}}, \\
& 0=\frac{d v}{d \eta}+\frac{1}{R_{m} \cos \theta} \frac{d^{2} B_{y}}{d \eta^{2}}-\frac{m}{R_{m}} \frac{d^{2} B_{x}}{d \eta^{2}}, \\
& -2 K^{2} v=\frac{d^{2} u}{d \eta^{2}}+\frac{M^{2} \cos \theta}{R_{m}} \frac{d B_{x}}{d \eta},
\end{aligned}
$$




$$
2 K^{2}(u-1)=\frac{d^{2} v}{d \eta^{2}}+\frac{M^{2} \cos \theta}{R_{m}} \frac{d B_{y}}{d \eta},
$$

where $K^{2}=\Omega L^{2} / v$ is rotation parameter, which is reciprocal of Ekmann number, $M^{2}=B_{0}{ }^{2} L^{2} \sigma / \rho v$ is magnetic parameter which is square of Hartmann number and $R_{m}=\sigma \mu_{e} U_{0} L$ is magnetic Reynolds number.

The boundary conditions ( $7 \mathrm{a})$ to (8b), in dimensionless form become

$$
\begin{aligned}
& u=0, v=0 \text { at } \eta=0 ; u=1, v=0 \text { at } \eta=1, \\
& B_{x}=B_{y}=0 \text { at } \eta=0 ; B_{x}=B_{y}=0 \text { at } \eta=1 .
\end{aligned}
$$

Combining equations (13) and (15) with (14) and (16) respectively, we obtain

$$
\begin{aligned}
& 0=\frac{d F}{d \eta}+\frac{1}{\cos \theta} \frac{d^{2} Q}{d \eta^{2}}-m i \frac{d^{2} Q}{d \eta^{2}}, \\
& 2 i K^{2}(F-1)=\frac{d^{2} F}{d \eta^{2}}+M^{2} \cos \theta \frac{d Q}{d \eta},
\end{aligned}
$$

where

$$
F=u+i v, Q=b_{x}+i b_{y}, b_{x}=B_{x} / R_{m} \text { and } b_{y}=B_{y} / R_{m} .
$$

The boundary conditions (17) and (18) in compact form become

$$
\begin{aligned}
& F=0 \text { at } \eta=0 ; F=1 \text { at } \eta=1, \\
& Q=0 \text { at } \eta=0 ; Q=0 \text { at } \eta=1 .
\end{aligned}
$$

Solution of equations (19) and (20) subject to the boundary conditions (21) and (22) is given by

$$
F(\eta)=\frac{1}{\lambda}[A \sinh \lambda \eta+B(\cosh \lambda \eta-1)]
$$

$Q(\eta)=\frac{1}{M^{2} \cos \theta}\left[\left(\frac{2 i K^{2}-\lambda^{2}}{\lambda^{2}}\right)\{A(\cosh \lambda \eta-1)+B \sinh \lambda \eta\}-\left(\frac{B}{\lambda}+1\right) 2 i k^{2} \eta\right]$,

where

$$
\begin{aligned}
& \lambda=\alpha+i \beta \\
& \alpha, \beta=\frac{1}{\sqrt{2}}\left[ \pm \frac{M^{2} \cos ^{2} \theta}{1+m^{2} \cos ^{2} \theta}+\left\{\frac{M^{4} \cos ^{4} \theta}{\left(1+m^{2} \cos ^{2} \theta\right)^{2}}+\left(2 K^{2}+\frac{M^{2} m \cos ^{3} \theta}{1+m^{2} \cos ^{2} \theta}\right)^{2}\right\}^{1 / 2}\right]^{1 / 2}, \\
& A=\left[\frac{\left(2 i K^{2}-\lambda^{2}\right) \lambda \sinh \lambda-2 i K^{2} \lambda^{2} \cosh \lambda}{2\left(2 i K^{2}-\lambda^{2}\right)(\cosh \lambda-1)-2 i K^{2} \lambda \sinh \lambda}\right], \\
& B=\left[\frac{2 i K^{2} \lambda^{2} \sinh \lambda-\left(2 i K^{2}-\lambda^{2}\right) \lambda(\cosh \lambda-1)}{2\left(2 i K^{2}-\lambda^{2}\right)(\cosh \lambda-1)-2 i K^{2} \lambda \sinh \lambda}\right] .
\end{aligned}
$$

\section{Particular Solutions}

We shall now derive few particular solutions from general solution (23) to (25)

1. Steady MHD Couette flow of class-II in a rotating system in the presence of an inclined magnetic field. Solution for this problem is obtained by setting $m=0$ in (23) to (25) which is given by

$$
\begin{aligned}
& F(\eta)=\frac{1}{\lambda_{1}}\left[A \sinh \lambda_{1} \eta+B\left(\cosh \lambda_{1} \eta-1\right)\right] \\
& Q(\eta)=\frac{1}{M^{2} \cos \theta}\left[\left(\frac{2 i K^{2}-\lambda_{1}^{2}}{\lambda_{1}^{2}}\right)\left\{A\left(\cosh \lambda_{1} \eta-1\right)+B \sinh \lambda_{1} \eta\right\}-\left(\frac{B}{\lambda_{1}}+1\right) 2 i K^{2} \eta\right],
\end{aligned}
$$

where

$$
\begin{aligned}
& \lambda_{1}=\alpha_{1}+i \beta_{1}, \\
& \alpha_{1}, \beta_{1}=\frac{1}{\sqrt{2}}\left[ \pm M^{2} \cos ^{2} \theta+\left(M^{4} \cos ^{4} \theta+4 K^{4}\right)^{1 / 2}\right]^{1 / 2},
\end{aligned}
$$




$$
\begin{aligned}
& A=\left[\frac{\left(2 i K^{2}-\lambda_{1}^{2}\right) \lambda_{1} \sinh \lambda_{1}-2 i K^{2} \lambda_{1}^{2} \cosh \lambda_{1}}{2\left(2 i K^{2}-\lambda_{1}^{2}\right)\left(\cosh \lambda_{1}-1\right)-2 i K^{2} \lambda_{1} \sinh \lambda_{1}}\right], \\
& B=\left[\frac{2 i K^{2} \lambda_{1}^{2} \sinh \lambda_{1}-\left(2 i K^{2}-\lambda_{1}^{2}\right) \lambda_{1}\left(\cosh \lambda_{1}-1\right)}{2\left(2 i K^{2}-\lambda_{1}^{2}\right)\left(\cosh \lambda_{1}-1\right)-2 i K^{2} \lambda_{1} \sinh \lambda_{1}}\right] .
\end{aligned}
$$

2. Steady MHD Couette flow of class-II in a rotating system in the presence of a uniform transverse magnetic field with Hall effects. Solution for this problem is obtained by setting $\theta=0$ in (23) to (25) which is given by

$$
\begin{aligned}
& F(\eta)=\frac{1}{\lambda_{2}}\left[A \sinh \lambda_{2} \eta+B\left(\cosh \lambda_{2} \eta-1\right)\right], \\
& Q(\eta)=\frac{1}{M^{2}}\left[\left(\frac{2 i K^{2}-\lambda_{2}^{2}}{\lambda_{2}{ }^{2}}\right)\left\{A\left(\cosh \lambda_{2} \eta-1\right)+B \sinh \lambda_{2} \eta\right\}-\left(\frac{B}{\lambda_{2}}+1\right) 2 i K^{2} \eta\right],
\end{aligned}
$$

where

$$
\begin{aligned}
& \lambda_{2}=\alpha_{2}+i \beta_{2}, \\
& \alpha_{2}, \beta_{2}=\frac{1}{\sqrt{2}}\left[ \pm \frac{M^{2}}{1+m^{2}}+\left\{\frac{M^{4}}{\left(1+m^{2}\right)^{2}}+\left(2 K^{2}+\frac{M^{2} m}{1+m^{2}}\right)^{2}\right\}^{1 / 2}\right]^{1 / 2}, \\
& A=\left[\frac{\left(2 i K^{2}-\lambda_{2}^{2}\right) \lambda_{2} \sinh \lambda_{2}-2 i K^{2} \lambda_{2}{ }^{2} \cosh \lambda_{2}}{2\left(2 i K^{2}-\lambda_{2}{ }^{2}\right)\left(\cosh \lambda_{2}-1\right)-2 i K^{2} \lambda_{2} \sinh \lambda_{2}}\right], \\
& B=\left[\frac{2 i K^{2} \lambda_{2}{ }^{2} \sinh \lambda_{2}-\left(2 i K^{2}-\lambda_{2}{ }^{2}\right) \lambda_{2}\left(\cosh \lambda_{2}-1\right)}{2\left(2 i K^{2}-\lambda_{2}{ }^{2}\right)\left(\cosh \lambda_{2}-1\right)-2 i K^{2} \lambda_{2} \sinh \lambda_{2}}\right] .
\end{aligned}
$$

3. Steady MHD Couette flow of class-II in a rotating system in the presence of a uniform transverse magnetic field. Solution for this problem is obtained by setting $m=0$ and $\theta=0$ in (23) to (25) which is given by

$$
\begin{aligned}
& F(\eta)=\frac{1}{\lambda_{3}}\left[A \sinh \lambda_{3} \eta+B\left(\cosh \lambda_{3} \eta-1\right)\right], \\
& Q(\eta)=\frac{1}{M^{2}}\left[\left(\frac{2 i K^{2}-\lambda_{3}^{2}}{\lambda_{3}{ }^{2}}\right)\left\{A\left(\cosh \lambda_{3} \eta-1\right)+B \sinh \lambda_{3} \eta\right\}-\left(\frac{B}{\lambda_{3}}+1\right) 2 i K^{2} \eta\right],
\end{aligned}
$$

where

$$
\begin{aligned}
& \lambda_{3}=\alpha_{3}+i \beta_{3}, \\
& \alpha_{3}, \beta_{3}=\frac{1}{\sqrt{2}}\left[ \pm M^{2}+\left(M^{4}+4 K^{4}\right)^{1 / 2}\right]^{1 / 2}, \\
& A=\left[\frac{\left(2 i K^{2}-\lambda_{3}^{2}\right) \lambda_{3} \sinh \lambda_{3}-2 i K^{2} \lambda_{3}^{2} \cosh \lambda_{3}}{2\left(2 i K^{2}-\lambda_{3}^{2}\right)\left(\cosh \lambda_{3}-1\right)-2 i K^{2} \lambda_{3} \sinh \lambda_{3}}\right], \\
& B=\left[\frac{2 i K^{2} \lambda_{3}^{2} \sinh \lambda_{3}-\left(2 i K^{2}-\lambda_{3}^{2}\right) \lambda_{3}\left(\cosh \lambda_{3}-1\right)}{2\left(2 i K^{2}-\lambda_{3}^{2}\right)\left(\cosh \lambda_{3}-1\right)-2 i K^{2} \lambda_{3} \sinh \lambda_{3}}\right] .
\end{aligned}
$$

This solution is in agreement with the solution obtained by Seth and Singh (2011).

\section{Shear stress at the plates}

Dimensionless shear stress $\tau_{x}$ and $\tau_{y}$ at the moving plate due to primary and secondary flow respectively are given by $\left(\tau_{x}+i \tau_{y}\right)_{\eta=1}=A \cosh \lambda+B \sinh \lambda$.

\section{Mass flow rates}

Dimensionless mass flow rates $Q_{x}$ and $Q_{y}$ in the primary and secondary flow directions respectively are given by

$$
Q_{x}+i Q_{y}=\frac{1}{\lambda^{2}}[A(\cosh \lambda-1)+B(\sinh \lambda-\lambda)] .
$$




\section{Asymptotic Solutions}

In order to gain some physical insight into the flow pattern, we shall now discuss asymptotic behavior of solution given by equations (23) to (25) for small and large values of $M^{2}$ and $K^{2}$.

Case I: When $M^{2}<<1$ and $K^{2}<<1$

Since $M^{2}$ and $K^{2}$ are very small, we neglect squares and higher powers of $M^{2}$ and $K^{2}$ and their product in equations (23) to (25) and we obtain components of velocity and induced magnetic field as

$$
\begin{aligned}
& u(\eta)=\eta+\frac{\eta}{12}\left(-1+3 \eta-2 \eta^{2}\right)\left(\frac{M^{2} \cos ^{2} \theta}{1+m^{2} \cos ^{2} \theta}\right)+\ldots . . \\
& v(\eta)=\frac{\eta}{6}\left(-1+3 \eta-2 \eta^{2}\right)\left[K^{2}\left(1+4 \eta-3 \eta^{2}\right)+\frac{1}{2}\left(\frac{M^{2} m \cos ^{3} \theta}{1+m^{2} \cos ^{2} \theta}\right)\right]+\ldots . \\
& b_{x}(\eta)=\left(\frac{\cos \theta}{1+m^{2} \cos ^{2} \theta}\right)\left[\frac{\eta(1-\eta)}{2}-\frac{M^{2} \cos ^{2} \theta\left(1-m^{2} \cos ^{2} \theta\right)}{24\left(1+m^{2} \cos ^{2} \theta\right)} \eta^{2}(1-\eta)^{2}-\frac{K^{2} m}{12} \eta\left(47-96 \eta+50 \eta^{2}-\eta^{3}\right)\right]+\ldots, \\
& b_{y}(\eta)=\left(\frac{\cos \theta}{1+m^{2} \cos ^{2} \theta}\right)\left[\frac{m \cos \theta}{2} \eta(1-\eta)-\frac{M^{2} m \cos ^{3} \theta}{12\left(1+m^{2} \cos ^{2} \theta\right)} \eta^{2}(1-\eta)^{2}+\frac{K^{2}}{12} \eta\left(47-96 \eta+50 \eta^{2}-\eta^{3}\right)\right]+\ldots .
\end{aligned}
$$

It is evident from the expressions (37) to (40) that in a slowly rotating system when the conductivity of the fluid is low primary velocity $u(\eta)$ is independent of rotation and secondary velocity $v(\eta)$, primary induced magnetic field $b_{x}(\eta)$ and secondary induced magnetic field $b_{y}(\eta)$ are affected by Hall current, magnetic field and rotation whereas fluid velocities and induced magnetic fields in both the directions are affected by angle of inclination of magnetic field $\theta$. In the absence of Hall current, secondary velocity $v(\eta)$ and secondary induced magnetic field $b_{y}(\eta)$ are unaffected by magnetic field whereas primary induced magnetic field $b_{x}(\eta)$ is unaffected by rotation.

Case II: When $K^{2}>>1$ and $M^{2} \sim O(1)$

When $K^{2}$ is large and $M^{2}$ is of small order of magnitude fluid flow becomes boundary layer type. For the boundary layer flow adjacent to the stationary plate $\eta=0$, we obtain velocities and induced magnetic fields from equations (23) to (25) as

$$
\begin{aligned}
& u(\eta)=1-e^{-\alpha_{4} \eta} \cos \left(\beta_{4} \eta\right), \\
& v(\eta)=e^{-\alpha_{4} \eta} \sin \left(\beta_{4} \eta\right), \\
& b_{x}(\eta)=\frac{\cos \theta}{2 K\left(1+m^{2} \cos ^{2} \theta\right)}\left[(1-m \cos \theta) e^{-\alpha_{4} \eta} \sin \beta_{4} \eta-(1+m \cos \theta)\left(e^{-\alpha_{4} \eta} \cos \beta_{4} \eta-1+\eta\right)\right], \\
& b_{y}(\eta)=\frac{\cos \theta}{2 K\left(1+m^{2} \cos ^{2} \theta\right)}\left[(1-m \cos \theta)\left(e^{-\alpha_{4} \eta} \cos \beta_{4} \eta-1+\eta\right)+(1+m \cos \theta) e^{-\alpha_{4} \eta} \sin \beta_{4} \eta\right],
\end{aligned}
$$

where

$$
\begin{aligned}
& \alpha_{4}=K\left[1+\frac{M^{2} \cos ^{2} \theta(m \cos \theta+1)}{4 K^{2}\left(1+m^{2} \cos ^{2} \theta\right)}\right], \\
& \beta_{4}=K\left[1+\frac{M^{2} \cos ^{2} \theta(m \cos \theta-1)}{4 K^{2}\left(1+m^{2} \cos ^{2} \theta\right)}\right] .
\end{aligned}
$$

It is evident from the equations (41) to (45) that there arises a thin boundary layer of thickness $O\left(\alpha_{4}^{-1}\right)$ near the stationary plate of the channel. This boundary layer may be identified as modified Ekman boundary layer and may be viewed as classical Ekman boundary layer modified by Hall current, magnetic field and angle of inclination of magnetic field. It is revealed from (45a) that $\alpha_{4}$ increases on increasing either $M^{2}$ or $K^{2}$. This implies that thickness of the boundary layer decreases on increasing either $M^{2}$ or $K^{2}$. Similar type of boundary layer arises near the moving plate of the channel. The exponential terms in the expressions (41) to (44) damped out quickly as $\eta$ increases. When $\eta \geq 1 / \alpha_{4}$ i.e. outside the boundary layer region, equations (41) to (44) reduce to 


$$
\begin{aligned}
& u(\eta) \approx 1, v(\eta) \approx 0, \\
& b_{x}(\eta) \approx \frac{-\cos \theta(1+m \cos \theta)}{2 K\left(1+m^{2} \cos ^{2} \theta\right)}(\eta-1), \quad b_{y}(\eta) \approx \frac{\cos \theta(1-m \cos \theta)}{2 K\left(1+m^{2} \cos ^{2} \theta\right)}(\eta-1) .
\end{aligned}
$$

Expressions in (46a) and (46b) demonstrate that, in the central core region, fluid flows in the primary flow direction only whereas induced magnetic fields persist in both the primary and secondary flow directions and vary linearly with $\eta$.

Case III: When $M^{2}>>1$ and $K^{2} \sim O(1)$

In this case also boundary layer type flow is expected. For the boundary layer flow near the stationary plate $\eta=0$, we obtain the components of velocity and induced magnetic field from the equations (23) to (25) as

$$
\begin{aligned}
& u(\eta)=\frac{1}{2}\left[\left(1+\frac{K^{2} c_{2}}{M \cos \theta}\right)\left(1-e^{-\alpha_{5} \eta} \cos \beta_{5} \eta\right)-\frac{K^{2} c_{1}}{M \cos \theta} e^{-\alpha_{5} \eta} \sin \beta_{5} \eta\right], \\
& v(\eta)=\frac{1}{2}\left[\left(1+\frac{K^{2} c_{2}}{M \cos \theta}\right) e^{-\alpha_{5} \eta} \sin \beta_{5} \eta+\frac{K^{2} c_{1}}{M \cos \theta}\left(1-e^{-\alpha_{5} \eta} \cos \beta_{5} \eta\right)\right], \\
& b_{x}(\eta)=\frac{1}{2 M^{2} \cos \theta}\left[\left\{\frac{c_{1} M \cos \theta}{\left(c_{1}{ }^{2}+c_{2}{ }^{2}\right)}\right\}\left(1-e^{-\alpha_{5} \eta} \cos \beta_{5} \eta\right)+\left\{3 K^{2}-\frac{c_{2} M \cos \theta}{\left(c_{1}{ }^{2}+c_{2}{ }^{2}\right)}\right\} e^{-\alpha_{5} \eta} \sin \beta_{5} \eta\right], \\
& b_{y}(\eta)=\frac{1}{2 M^{2} \cos \theta}\left[\left\{\frac{c_{2} M \cos \theta}{\left(c_{1}{ }^{2}+c_{2}{ }^{2}\right)}-3 K^{2}\right\}\left(1-e^{-\alpha_{5} \eta} \cos \beta_{5} \eta\right)+\left\{\frac{c_{1} M \cos \theta}{\left(c_{1}{ }^{2}+c_{2}{ }^{2}\right)}\right\} e^{-\alpha_{5} \eta} \sin \beta_{5} \eta+2 K^{2} \eta\right],
\end{aligned}
$$

where

$$
\begin{aligned}
& \alpha_{5}=\frac{M \cos \theta}{\sqrt{\left(1+m^{2} \cos ^{2} \theta\right)}}\left[c_{1}\left(1+\frac{m K^{2}}{M^{2} \cos \theta}\right)-\frac{c_{2} K^{2}}{M^{2} \cos ^{2} \theta}\right], \\
& \beta_{5}=\frac{M \cos \theta}{\sqrt{\left(1+m^{2} \cos ^{2} \theta\right)}}\left[c_{2}\left(1+\frac{m K^{2}}{M^{2} \cos \theta}\right)+\frac{c_{1} K^{2}}{M^{2} \cos ^{2} \theta}\right], \\
& c_{1}=\frac{1}{\sqrt{2}}\left[1+\left(1+m^{2} \cos ^{2} \theta\right)^{\frac{1}{2}}\right]^{\frac{1}{2}}, \\
& c_{2}=\frac{1}{\sqrt{2}}\left[-1+\left(1+m^{2} \cos ^{2} \theta\right)^{\frac{1}{2}}\right]^{\frac{1}{2}} .
\end{aligned}
$$

It is revealed from the expressions (47) to (51) that there appears a thin boundary layer of thickness $O\left(\alpha_{5}^{-1}\right)$ near the stationary plate of the channel. This boundary layer may be recognized as modified Hartmann boundary layer and may be viewed as classical Hartmann boundary layer modified by Hall current, rotation and angle of inclination of magnetic field. The thickness of this boundary layer decreases on increasing $M^{2}$. In the absence of Hall current there arises a thin boundary layer of thickness $O(1 / M \cos \theta)$ near the stationary plate. Similar type of boundary layer arises near moving plate of the channel. When $\eta \geq 1 / \alpha_{5}$ i.e. outside the boundary layer region, solution (47) to (50) assumes the form

$$
\begin{aligned}
& u(\eta) \approx \frac{1}{2}\left(1+\frac{K^{2} c_{2}}{M \cos \theta}\right), v(\eta) \approx \frac{K^{2} c_{1}}{M \cos \theta}, \\
& b_{x}(\eta) \approx \frac{1}{2 M^{2} \cos \theta}\left[\frac{c_{1} M \cos \theta}{\left(c_{1}^{2}+c_{2}^{2}\right)}+3 K^{2}\right] \text {, } \\
& b_{y}(\eta) \approx \frac{1}{2 M^{2} \cos \theta}\left[\frac{c_{2} M \cos \theta}{\left(c_{1}^{2}+c_{2}^{2}\right)}-3 K^{2}+2 K^{2} \eta\right] \text {. }
\end{aligned}
$$


Expressions in (52a) to (52c) show that, in the central core region, fluid velocities and induced magnetic fields persist in both the directions and are affected by Hall current, magnetic field, rotation and angle of inclination of magnetic field.

It was noticed that in the absence of Hall current and angle of inclination of magnetic field, the results obtained in cases I to III are in agreement with the results obtained by Seth and Singh (2011).

\section{Heat Transfer Characteristics}

We shall now discuss heat transfer characteristics of this fluid flow problem when the stationary and moving plates of the channel are maintained at uniform temperatures $T_{0}$ and $T_{1}$ respectively, where $T_{0}<T^{\prime}<T_{1}, T^{\prime}$ being the fluid temperature.

Energy equation taking viscous and Joule dissipations into account is given by

$$
\alpha * \frac{d^{2} T^{\prime}}{d z^{2}}+\frac{v}{C_{p}}\left[\left(\frac{d u_{x}}{d z}\right)^{2}+\left(\frac{d u_{y}}{d z}\right)^{2}\right]+\frac{1}{\sigma \rho C_{p}}\left[\left(\frac{d B_{x}{ }^{\prime}}{d z}\right)^{2}+\left(\frac{d B_{y}{ }^{\prime}}{d z}\right)^{2}\right]=0,
$$

where $\alpha^{*}$ and $C_{p}$ are thermal diffusivity and specific heat at constant pressure respectively.

Boundary conditions for the temperature field are

$$
T^{\prime}=T_{0} \text { at } z=0 \quad \text { and } \quad T^{\prime}=T_{1} \text { at } z=L \text {. }
$$

Equation (53), in dimensionless form, become

$$
\frac{d^{2} T}{d \eta^{2}}+P_{r} E_{r}\left[\left\{\left(\frac{d u}{d \eta}\right)^{2}+\left(\frac{d v}{d \eta}\right)^{2}\right\}+M^{2}\left\{\left(\frac{d b_{x}}{d \eta}\right)^{2}+\left(\frac{d b_{y}}{d \eta}\right)^{2}\right\}\right]=0,
$$

where $T=\frac{T^{\prime}-T_{0}}{T_{1}-T_{0}}, P_{r}=\frac{v}{\alpha^{*}}$ and $E_{r}=\frac{U_{0}{ }^{2}}{C_{p}\left(T_{1}-T_{0}\right)}$ are dimensionless fluid temperature, Prandtl number and Eckert number respectively.

Boundary conditions (54), dimensionless form, assume the form

$$
T(0)=0 \text { and } T(1)=1 \text {. }
$$

Making use of solution (23) to (25) in equation (55), the resulting differential equation subject to boundary conditions (56) is solved numerically with the help of MATLAB software. The numerical values of rate of heat transfer at the stationary and moving plates are also computed with the help of MATLAB software.

\section{Results and discussion}

Effects of magnetic field and rotation on hydromagnetic Couette flow of class-II of a viscous, incompressible and electrically conducting fluid in a rotating system with electrically non-conducting walls in the presence of a uniform transverse magnetic field is investigated by Seth and Singh (2011). To study the effects of Hall current and angle of inclination of magnetic field on flowfield, the numerical values of primary and secondary fluid velocities and primary and secondary induced magnetic fields, computed from analytical solution (23) to (25) by MATLAB software, are displayed graphically versus channel width variable $\eta$ in figures 2 to 5 for various values of Hall current parameter $m$ and angle of inclination of magnetic field $\theta$ taking magnetic parameter $M^{2}=20$ and rotation parameter $K^{2}=3$. It is evident from figure 2 that the primary velocity $u$ increases in the upper half of the channel and decreases in the lower half of the channel whereas secondary velocity $v$ decreases in the upper half of the channel and increases in the lower half of the channel on increasing $m$ which implies that Hall current tends to accelerate fluid flow in the primary flow direction in the upper half of the channel and it tends to retard fluid flow in the primary flow direction in the lower half of the channel whereas it has reverse effect on the fluid flow in secondary flow direction. It may be noted that, in general, in MHD Couette flow of class-I, Hall current tends to induce cross flow i.e. secondary flow by suppressing primary flow. However, above trend which is observed in figure 2 is different than the trend observed in MHD Couette flow of class-I. This is due to reason that the effect of non- zero pressure gradient term, which is given by equation (9b), is considered in this fluid flow problem. It was noticed from figure 3 that primary velocity $u$ increases in the upper half of the channel and decreases in the lower half of the channel whereas secondary velocity $v$ increases throughout the channel on increasing $\theta$ which implies that the angle of inclination of magnetic field tends to accelerate fluid flow in the primary flow direction in the upper half of the channel and it has reverse effect on the fluid flow in the primary flow direction in the lower half of the channel. Angle of inclination of magnetic field tends to accelerate fluid flow in the secondary flow direction throughout the channel. It is seen from figure 4 that primary induced magnetic field $b_{x}$ decreases whereas secondary induced magnetic field $b_{y}$ increases on increasing $m$ which implies that Hall 
current tends to reduce primary induced magnetic field whereas it has reverse effect on secondary induced magnetic field. It is evident from figure 5 that both the primary and secondary induced magnetic fields decrease on increasing $\theta$ which implies that angle of inclination of magnetic field tends to reduce both the primary and secondary induced magnetic fields.

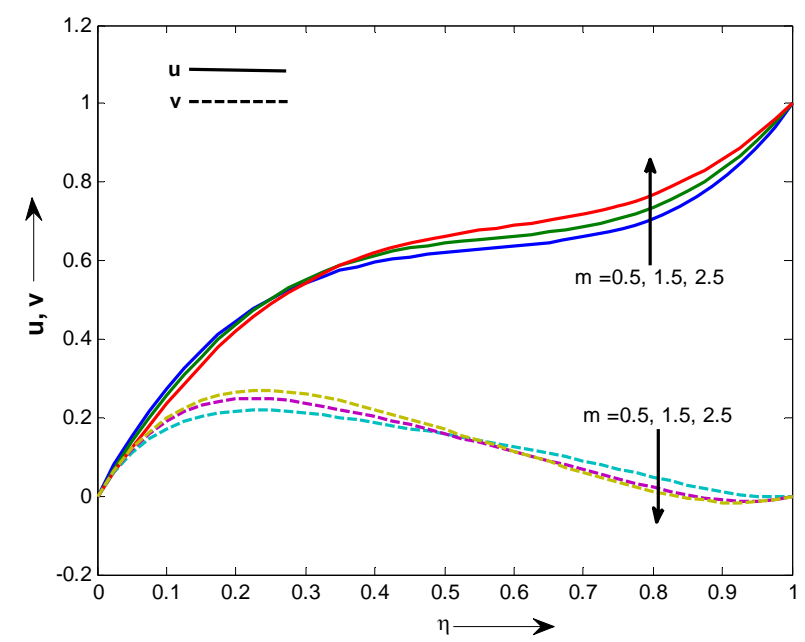

Figure 2 Velocity profiles when $\theta=\pi / 4$.

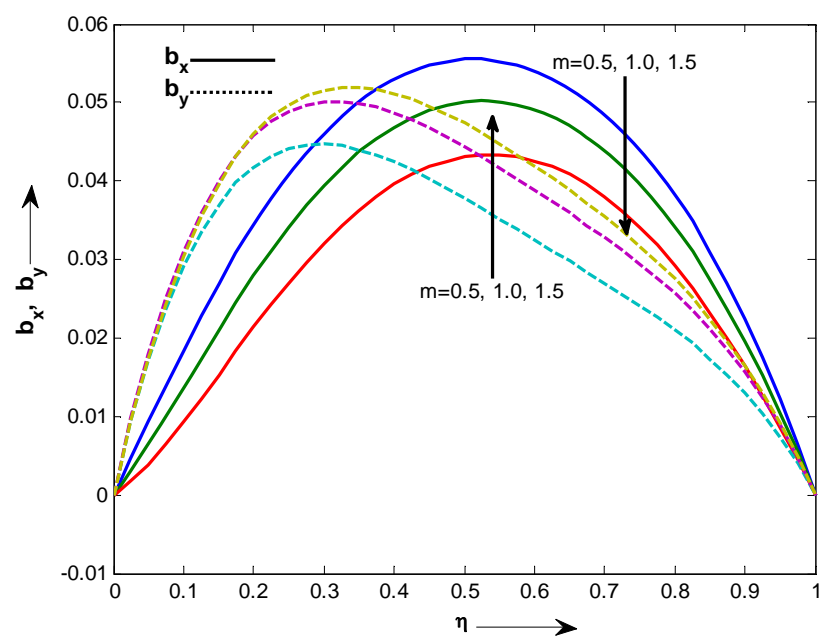

Figure 4 Induced Magnetic field profiles when $\theta=\pi / 4$.

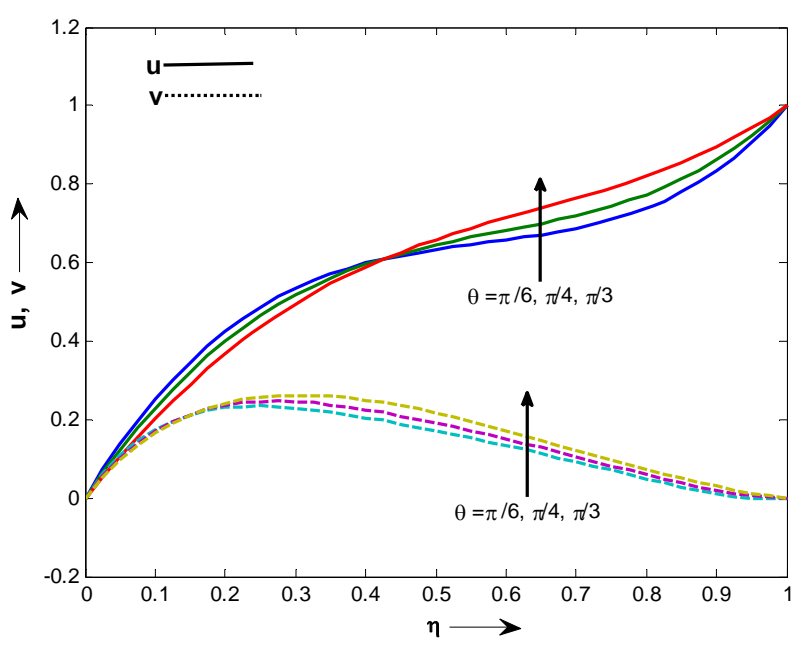

Figure 3 Velocity profiles when $m=0.5$.

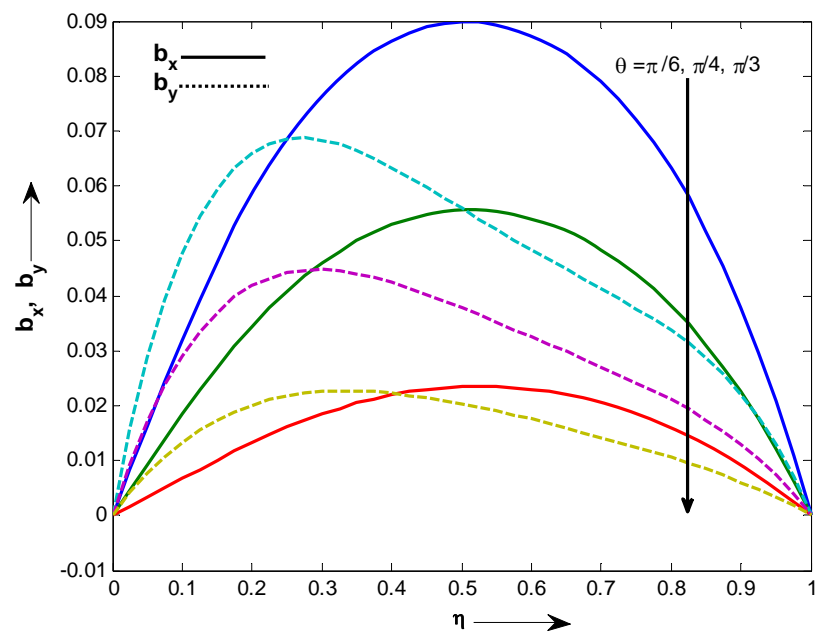

Figure 5 Induced Magnetic field profiles when $m=0.5$. 




Figure 6 Temperature profiles when $\theta=\pi / 4$.

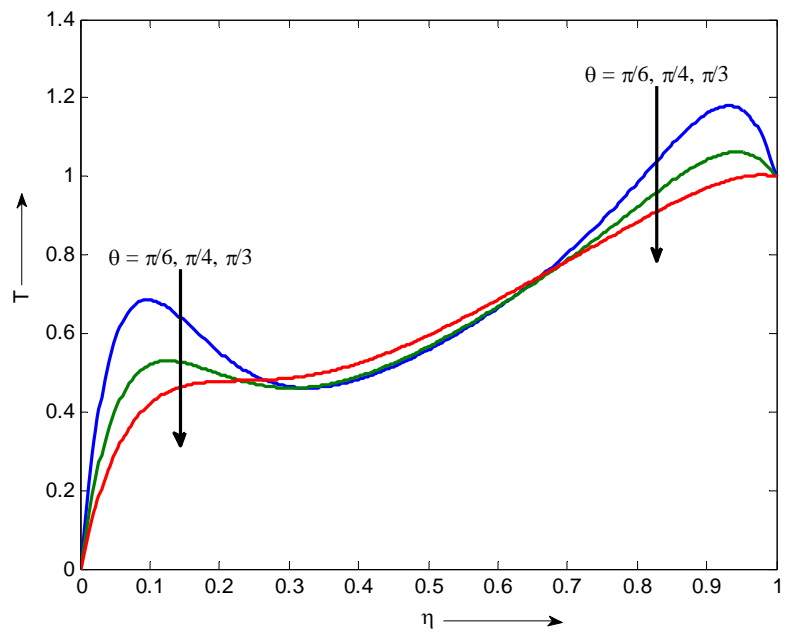

Figure 7 Temperature profiles when $m=0.5$.

To study the effects of Hall current and angle of inclination of magnetic field on fluid temperature, the numerical solution of energy equation, computed with the help of MATLAB software, is depicted graphically versus channel width variable $\eta$ in figures 6 and 7 for various values of $m$ and $\theta$ taking $M^{2}=20, K^{2}=3, P_{r}=0.71$ and $E_{r}=2$. It is seen from figures 6 and 7 that fluid temperature $T$ decreases in the regions near the stationary and moving plates of the channel on increasing either $m$ or $\theta$ which implies that Hall current and angle of inclination of magnetic field have tendency to reduce fluid temperature in the regions near the stationary and moving plates of the channel.

The numerical values of primary and secondary shear stress at the moving plate and that of primary and secondary mass flow rates, computed from the analytical expressions (35) and (36) by MATLAB software, are presented in tabular form in tables 1and 2 for various values of $m$ and $\theta$ taking $M^{2}=20$ and $K^{2}=3$. It is evident from table 1 that primary shear stress at the moving plate i.e. $\left(\tau_{x}\right)_{\eta=1}$ decreases on increasing either $m$ or $\theta$. Secondary shear stress at the moving plate i.e. $\left(\tau_{y}\right)_{\eta=1}$ decreases, attains a minimum and then increases in magnitude on increasing $\theta$. With an increase in $m,\left(\tau_{y}\right)_{\eta=1}$ increases when $\theta=\pi / 6$, it decreases in magnitude, attains a minimum and then increases when $\theta=\pi / 4$ and it decreases in magnitude when $\theta=\pi / 3$. This implies that angle of inclination of magnetic field and Hall current have tendency to reduce primary shear stress at the moving plate. There exists flow separation at the moving plate in the secondary flow direction on increasing $\theta$ for every value of $m$ or on increasing $m$ when $\theta=\pi / 4$. It is obsorved from table 2 that primary mass flow rate i.e. $Q_{x}$ and secondary mass flow rate i.e. $Q_{y}$ increase on increasing $\theta . Q_{x}$ increases, attains a maximum and then decreases on increasing $m$. With theincreasein $m, Q_{y}$ increases, attains a maximum and then decreases when $\theta=\pi / 6$, it decreases when $\theta=\pi / 4$ and it decreases, attains a minimum and then increases when $\theta=\pi / 3$. This implies that angle of inclination of magnetic field tends to enhance both the primary and secondary mass flow rates.

Table 1 Primary and secondary shear stress at moving plate.

\begin{tabular}{|c|c|c|c|c|c|c|}
\hline \multirow{2}{*}{$\theta \rightarrow$} & \multicolumn{3}{|c|}{$\left(\tau_{x}\right)_{\eta=1}$} & \multicolumn{3}{c|}{$\left(\tau_{y}\right)_{\eta=1}$} \\
\cline { 2 - 7 }$m \downarrow$ & $\pi / 6$ & $\pi / 4$ & $\pi / 3$ & $\pi / 6$ & $\pi / 4$ & $\pi / 3$ \\
\hline $\mathbf{0 . 5 0}$ & 2.1300 & 1.7277 & 1.2396 & 0.1616 & -0.0065 & -0.1770 \\
\hline $\mathbf{1 . 0 0}$ & 1.9076 & 1.6128 & 1.2167 & 0.3258 & 0.1396 & -0.0797 \\
\hline $\mathbf{1 . 5 0}$ & 1.6765 & 1.4657 & 1.1653 & 0.3789 & 0.2059 & -0.0180 \\
\hline
\end{tabular}


Table 2 Primary and secondary mass flow rates.

\begin{tabular}{|c|c|c|c|c|c|c|}
\hline \multirow{2}{*}{$\theta \rightarrow$} & \multicolumn{3}{|c|}{$Q_{x}$} & \multicolumn{3}{c|}{$Q_{y}$} \\
\cline { 2 - 7 }$m \downarrow$ & $\pi / 6$ & $\pi / 4$ & $\pi / 3$ & $\pi / 6$ & $\pi / 4$ & $\pi / 3$ \\
\hline $\mathbf{0 . 5 0}$ & 0.5871 & 0.5931 & 0.6013 & 0.1315 & 0.1420 & 0.1552 \\
\hline $\mathbf{1 . 0 0}$ & 0.6009 & 0.6030 & 0.6062 & 0.1339 & 0.1417 & 0.1528 \\
\hline $\mathbf{1 . 5 0}$ & 0.5947 & 0.5984 & 0.6038 & 0.1314 & 0.1407 & 0.1533 \\
\hline
\end{tabular}

The numerical values of rate of heat transfer at the stationary and moving plates of the channel are computed with the help of MATLAB software and are presented in table 3 for various values of $m$ and $\theta$ taking $M^{2}=20, K^{2}=3, P_{r}=0.71$ and $E_{r}=2$. It is found from table 3 that rate of heat transfer at the stationary plate i.e. $\left(\frac{d T}{d \eta}\right)_{\eta=0}$ and rate of heat transfer at the moving plate i.e. $\left(\frac{d T}{d \eta}\right)_{\eta=1}$ decrease on increasing $\theta \cdot\left(\frac{d T}{d \eta}\right)_{\eta=0}$ decreases on increasing $m$ when $\theta=\pi / 6$ and $\pi / 4$ whereas $\left(\frac{d T}{d \eta}\right)_{\eta=1}$ decreases on increasing $m$. This implies that angle of inclination of magnetic field tends to reduce rate of heat transfer at both the stationary and moving plates of the channel. Hall current tends to reduce rate of heat transfer at the moving plate of the channel and it tends to reduce rate of heat transfer at the stationary plate of the channel when $\theta \leq \pi / 4$.

Table 3 Rate of heat transfer at the stationary and moving plates.

\begin{tabular}{|c|c|c|c|c|c|c|}
\hline \multirow{2}{*}{$\theta \rightarrow$} & \multicolumn{3}{|c|}{$-\left(\frac{d T}{d \eta}\right)_{\eta=0}$} & \multicolumn{3}{c|}{$-\left(\frac{d T}{d \eta}\right)_{\eta=1}$} \\
\cline { 2 - 7 }$m \downarrow$ & $\pi / 6$ & $\pi / 4$ & $\pi / 3$ & $\pi / 6$ & $\pi / 4$ & $\pi / 3$ \\
\hline $\mathbf{0 . 5 0}$ & 20.2005 & 12.7084 & 8.3112 & 23.4908 & 11.5133 & 5.0256 \\
\hline $\mathbf{1 . 0 0}$ & 18.4450 & 12.5301 & 8.5132 & 19.0866 & 10.3628 & 4.8986 \\
\hline $\mathbf{1 . 5 0}$ & 16.1880 & 11.8257 & 8.5019 & 14.8089 & 8.8091 & 4.6074 \\
\hline
\end{tabular}

The effects of Hall current and angle of inclination of magnetic field, which are observed in figures 2 to 7 , are due to presence of non-zero pressure gradient term in the governig equations for fluid flow problem. It is noticed from equations (5), (6), (10) and (11) that there is no interaction between fluid flow and applied magnetic field when $\theta=\pi / 2$. This fluid flow problem reduces to MHD Couette flow of class-II in a rotating system in the presence of a uniform transverse magnetic field when $\theta=0$. We have already provided the solution of this problem as a particular solution (29) to (31). In the present problem angle of inclination of magnetic field $\theta$ lies between 0 and $\pi / 2$. The numerical values of fluid velocity, induced magnetic field, fluid temperature, shear stress at the moving plate, mass flow rate and rate of heat transfer at both the plates indicate that there is an effective interaction between fluid flow and magnetic field when $\theta$ is very small. However, in the problems of geophysical and astrophysical interest value of angle $\theta$ depends on the position of magnetic field. Due to this reason we have computed numerical results for fluid velocity, induced magnetic field, fluid temperature, shear stress at the moving plate, mass flow rate and rate of heat transfer at both the plates when $\theta=\pi / 6, \pi / 4$ and $\pi / 3$. To the authors' knowledge such type of fluid flow is not considered in literature.

\section{Conclusion}

The present investigation deals with the theoretical study of Hall effects on hydromagnetic Couette flow of class-II in a rotating system in the presence of an inclined magnetic field with asymmetric heating /cooling of the walls. The significant results are summerized below

(i) Hall current tends to accelerate fluid flow in the primary flow direction in the upper half of the channel and it tends to retard fluid flow in the primary flow direction in the lower half of the channel whereas it has reverse effect on the fluid flow in secondary flow direction.

(ii) Angle of inclination of magnetic field tends to accelerate fluid flow in the primary flow direction in the upper half of the channel and it has reverse effect on the fluid flow in the primary flow direction in the lower half of the 
channel. Angle of inclination of magnetic field tends to accelerate fluid flow in the secondary flow direction throughout the channel.

(iii) Hall current tends to reduce primary induced magnetic field whereas it has reverse effect on secondary induced magnetic field.

(iv) Angle of inclination of magnetic field tends to reduce both the primary and secondary induced magnetic fields.

(v) Hall current and angle of inclination of magnetic field have tendency to reduce fluid temperature in the regions near the stationary and moving plates of the channel.

(vi) Angle of inclination of magnetic field and Hall current have tendency to reduce primary shear stress at the moving plate. There exists flow separation at the moving plate in the secondary flow direction on increasing $\theta$ for every value of $m$ or on increasing $m$ when $\theta=\pi / 4$.

(vii) Angle of inclination of magnetic field tends to enhance both the primary and secondary mass flow rates.

(viii) Angle of inclination of magnetic field tends to reduce rate of heat transfer at both the stationary and moving plates. Hall current tends to reduce rate of heat transfer at the moving plate of the channel and it tends to reduce rate of heat transfer at the stationary plate of the channel when $\theta \leq \pi / 4$.

Present investigation will help to find the angle(s) at which the system gives the best performance with respect to the maximum and minimum values of major quantities viz. magnetic field, Hall current and rotation by using an optimization technique, namely, Genatic Algorithm Technique. This will be a topic of research in the future.

\section{Acknowledgement}

Authors are grateful to the referees for their valuable suggestions and comments which help them to improve the quality of this research paper.

\section{References}

Batchelor, G. K. 1988. An Introduction to Fluid Dynamics. Combridge Univ. Press, Cambridge (U.K).

Bég, O. A., Sim, L., Zueco, J. and Bhargava, R. 2010. Numerical study of magnetohydrodynamic viscous plasma flow in rotating porous media with Hall currents and inclined magnetic field influence. Comm. Nonlinear Sci. Num. Simu., Vol. 15, pp. 345-359.

Chandran, P., Sacheti, N. C. and Singh, A. K. 1993. Effects of rotation on unsteady hydromagnetic Couette Flow, J. Astrophys. Space Sci., Vol. 202, pp. 1-10.

Chauhan, D. S. and Agrawal, R. 2012. Effects of Hall current on MHD Couette flow in a channel partially filled with porous medium in a rotating system. Meccanica., Vol. 47, pp. 405-421.

Cramer, R. K. and Pai, S. I. 1973. Magnetofluid dynamics for engineers and applied physcists. McGraw Hill Book Company, New York.

Das, B. K., Guria, M. and Jana, R. N. 2008. Unsteady Couette flow in a rotating system. Meccanica, Vol. 43, pp. 517-521.

Ganapathy, R. 1994. A note on oscillatory Couette flow in a rotating system. Trans. ASME J. Appl. Mech., Vol. 61, pp. 208-209.

Guchhait, S., Das, S., Jana, R. N. and Ghosh, S. K. 2011. Combined effets of Hall current and rotation on unsteady Couette flow in porous channel. World J. Mech., Vol. 1. pp. 87-99.

Guria, M., Das, S., Jana, R. N. and Ghosh, S. K. 2009. Oscillatory Couette flow in the presence inclined magnetic field. Meccanica, Vol. 44, pp. 555-564.

Hayat, T., Nadeem, S. and Asghar, S. 2004a. Hydromagnetic Couette flow of a Oldroyd-B fluid in a rotating system. Int. J. Engng. Sci., Vol. 42, pp.65-78.

Hayat, T., Nadeem, S., Siddiqui, A. M. and Asghar, S. 2004b. An oscillatory hydromagnetic non-newtonian in a rotating system. Appl. Math. Lett., Vol. 17, pp. 609-614.

Jha, B. K. and Apere, C. A. 2011. Hall and ion-slip effects on unsteady MHD Couette flow in a rotating system with suction and injection, J. Phys. Soc. Japan, 80, DOI:10.1143/JPSJ.80.114401.

Jana, R. N., Datta, N. and Mazumder, B. S. 1977. Magnetohydrodynamic Couette flow and heat transfer in a rotating system. J. Phys. Soc. Japan, Vol. 42, pp. 1034-1039.

Mazumder, B. S. 1991. An exact solution of oscillatory Couette flow in a rotating system. Trans. ASME J. Appl. Mech., Vol. 56, pp. 1104-1107.

Prasad, B. G. and Kumar, R. 2012. Unsteady hydromagnetic Couette flow through a porous medium in a rotating system, Theo. Appl. Mech. Letters, Vol. 1. DOI: 10.1063/2.1104205.

Seth, G. S. and Maiti, M. K. 1982. MHD Couette flow and heat transfer in a rotating system. Ind. J. Pure and Appl. Math., Vol. 13, pp. 931-945. 
Seth, G. S., Hussain, S. M. and Singh, J. K. 2011. MHD Couette flow of class-II in a rotating system. J. Appl. Math. \& Bioinformatics, Vol. 1, No. 1, pp.31-54.

Seth, G. S. and Singh, J. K. 2011. Steady hydromagnetic Couette flow in a rotating system with non-conducting walls. Int. J. Eng. Sci. and Tech.., Vol. 3, No. 2, pp. 146-156.

Seth, G. S., Nandkeolyar, R. and Ansari, Md. S. 2012. Effects of Hall current and rotation on unsteady MHD Couette flow in the presence of an inclined magnetic field, J. Appl. Fluid Mech., Vol. 5, No. 2, pp. 67-74.

Seth, G. S. and Singh, J. K. (2012). Unsteady MHD Couette flow of class-II of a viscous incompressible electrically conducting fluid in a rotating system, Int. J. Appl. Mech. Eng., Vol. 17, No. (2/3).(To appear in 2012).

Sherman, A. and Sutton, G. W. 1962. Magnetohydrodynamics. North Western Univ. Press, Evanston.

Singh, A. K., Sacheti, N.C. and Chandran, P. 1994. Transient effects on magnetohydrodynamic Couette flow with rotation, Int. J. Engng. Sci., Vol. 32, pp.133-139.

Singh, K. D. 2000. An oscillatory Couette flow in a rotating system. ZAMM, Vol. 8, pp. 429-432.

Sutton, G. W. and Sherman, A. 1965. Engineering Magnetohydrodynamics. McGraw-Hill, New York.

\section{Biographical notes}

Dr. G. S. Seth is a Professor in the Department of Applied Mathematics, Indian School of Mines, Dhanbad, India. He received his Ph. D. in the Mathematics from Indian Institute of Technology, Kharagpur, India. He has more than thirty three years of experience of teaching and research. His current area of research studies includes Fluid dynamics, Magnetohydrodynamics and heat transfer. He was visiting Assistant Professor at University of Aden, Republic of Yemen during the period September 01, 1991 to August 31, 1993. He has published more than sixty three research papers in national/International journals of repute.

S. M. Hussain received M. Sc. Degree in Mathematics from Vinova Bhave University, Hazaribag, Jharkhand, India in 2008. He received M. Phil degree in Applied Mathematics from Indian School of Mines, Dhanbad in 2009. He joined O. P. Jindal Institute of Technology, Raigarh, India as an Assistant Professor in the Department of Mathematics in 2009. In the year 2011, he has joined as a part time research scholar in the Department of Applied Mathematics to do the research work leading to $\mathrm{Ph}$. D. degree on the research topic entitled "Investigation of Some Problems of Fluid Flow and Heat Transfer in Magnetohydrodynamics”. He has three years of teaching experience and 2 years of research experience.

Received November 2011

Accepted May 2012

Final acceptance in revised form June 2012 